Ismail Moumouni, Aurelle de Romemont, Fortuné Amonsou-Biaou et Guy Faure

\title{
Standardisation du conseil agricole et diversité des modalités d'action des conseillers au Bénin
}

\begin{abstract}
Avertissement
Le contenu de ce site relève de la législation française sur la propriété intellectuelle et est la propriété exclusive de l'éditeur.

Les œuvres figurant sur ce site peuvent être consultées et reproduites sur un support papier ou numérique sous réserve qu'elles soient strictement réservées à un usage soit personnel, soit scientifique ou pédagogique excluant toute exploitation commerciale. La reproduction devra obligatoirement mentionner l'éditeur, le nom de la revue, l'auteur et la référence du document.

Toute autre reproduction est interdite sauf accord préalable de l'éditeur, en dehors des cas prévus par la législation en vigueur en France.
\end{abstract}

\section{revues.org}

Revues.org est un portail de revues en sciences humaines et sociales développé par le Cléo, Centre pour l'édition électronique ouverte (CNRS, EHESS, UP, UAPV).

\begin{abstract}
Référence électronique
Éditeur : Société Française d'Économie rurale

http://economierurale.revues.org

http://www.revues.org

Document accessible en ligne sur : http://economierurale.revues.org/4691

Ce document est le fac-similé de l'édition papier.

Cet article a été téléchargé sur le portail Cairn (http://www.cairn.info).
\end{abstract}

Ismail Moumouni, Aurelle de Romemont, Fortuné Amonsou-Biaou et Guy Faure, «Standardisation du conseil agricole et diversité des modalités d'action des conseillers au Bénin », Économie rurale [En ligne], 348 | juillet-août 2015, mis en ligne le 01 janvier 2017, consulté le 18 septembre 2015. URL : http://economierurale.revues.org/4691

\section{CAIR N}

Chercher. Repérer. Avancer.

Distribution électronique Cairn pour Société Française d'Économie rurale et pour Revues.org (Centre pour l'édition électronique ouverte)

(c) Tous droits réservés 


\title{
Standardisation du conseil agricole et diversité des modalités d'action des conseillers au Bénin
}

\author{
Ismail MOUMOUNI • Faculté d'Agronomie, Université de Parakou, Bénin \\ ismailmm@gmail.com \\ Aurelle de ROMEMONT • UMR Innovation, CIRAD-INRA-Supagro, Montpellier \\ Fortuné AMONSOU-BIAOU • Faculté d'Agronomie, Université de Parakou, Bénin \\ Guy FAURE • UMR Innovation, CIRAD-INRA-Supagro, Montpellier
}

Cet article analyse l'influence de la représentation des conseillers agricoles sur la mise en œuvre du conseil en se basant sur une étude de cas au Bénin. En analysant les profils professionnels des conseillers, leurs représentations de leurs rôles et les activités qu'ils mènent avec des groupes d'agriculteurs, nous avons identifié trois types de conseillers. II s'agit des conseillers « vulgarisant des techniques agricoles et de concepts de gestion », des conseillers " en appui à la gestion et aidant à la décision » et des conseillers " en appui à la gestion, en intermédiation et facilitation ». Ces résultats permettent de comprendre comment, dans les mêmes conditions professionnelles et en mobilisant une méthode standardisée de conseil, les conseillers peuvent s'inscrire dans des registres d'action différents.

MOTS-CLÉS : apprentissage, Bénin, conseil à l'exploitation familiale, conseiller, représentation

\section{Standardization of management advice to family farms and diversity of the modalities of action of advisers in Benin}

This paper analyzes the influence of the perception of farm advisers' on the implementation of advice. It is based on a case study in Benin. Through analyzing professional profiles of advisers, their perception of their roles, and the activities that they conducted, we identified three types of advisers. There are advisers "disseminating agricultural techniques and management concepts", advisers "supporting management and decision-making" and advisers "supporting management, intermediation and facilitation". These results highlighted how, in the same professional conditions and when using a standardized advisory method, advisers can operate differently. (JEL: Q16)

KEYWORDS: learning, Benin, management advice to family farm, adviser, perception

e rôle des conseillers agricoles en
Afrique de l'Ouest a évolué au cours
du temps. Pendant plusieurs décennies,
les conseillers avaient une activité visant
essentiellement à faciliter le transfert de
technologies et de connaissances, basée
sur le modèle de « diffusion des innova-
tions » (Rogers, 1988). Face à un échec de
ce modèle pour résoudre des problèmes
complexes, certaines organisations de
conseil ont cherché à adapter le contenu
du message des conseillers à la diversité
des situations (Djamen et al., 2010). Cette évolution s'est traduite par une diversification croissante des profils de conseillers (Faure et al., 2012). Une première classe de conseillers spécialisés vise à résoudre des problèmes spécifiques (conseil technique, conseil juridique, etc.). Ils travaillent plutôt dans le secteur privé (firmes de l'aval ou de l'amont, bureau d'étude...). Une deuxième classe de conseillers est porteuse de compétences plus diversifiées, afin de résoudre un plus large spectre de problèmes (gestion de l'exploitation, développement de projets territoriaux, etc.). Ils 
travaillent plutôt dans le secteur public, les Organisations non gouvernementales (ONG) ou les organisations de producteurs. Dans ce contexte, les relations entre les conseillers et les agriculteurs ont également évolué. Dans le cadre d'un conseil technique spécialisé, la relation de conseil reste souvent de nature prescriptive. On observe cependant une reconnaissance croissante par les acteurs du conseil de la nécessité de promouvoir une co-construction des problèmes et des solutions entre le conseiller et l'agriculteur (Cerf et Hemidy, 1999 ; Ingram, 2008). Le conseil, vu comme un service immatériel, interroge la diversité des formes d'interactions entre fournisseurs et clients constitutives de la relation de service (Gadrey, 1994 ; Labarthe et Laurent, 2011). Cependant, cette diversité des relations de conseil n'exclut pas la standardisation des méthodes de conseil par les organisations de conseil afin de rationaliser les activités de leurs conseillers. Cette standardisation du service peut être vue comme le mécanisme par lequel un prestataire réussit à fournir dans les mêmes conditions, des services de même nature et de même qualité, à un public plus large. La mise en œuvre d'un conseil qui vise à co-construire les problèmes et les solutions, peut donc se concevoir dans le cadre de méthode de conseil standardisée. Dans une logique de conseil standardisé, les conseillers sont invités à mettre en œuvre sur le terrain un cahier des charges élaboré par l'organisation de conseil qui définit les manières de faire et les marges de manœuvre pour adapter le service à la diversité des demandes des agriculteurs.

Cependant, les conseillers ont des conceptions diversifiées de leurs activités (Lemery, 1994). Ainsi, des différences importantes s'observent entre conseillers d'une même organisation dans la mise en œuvre du conseil. Andersen (2004) identifie, à partir d'une étude de cas au Danemark, trois profils différents de conseillers au sein d'une même organisation de conseil. Ils diffèrent en fonction de la relation de conseil qu'ils développent : le spécialiste qui délivre son conseil sans interagir avec le producteur ; le spécialiste qui interagit avec le producteur pour adapter son conseil ; le conseiller qui écoute et interagit avec le producteur pour construire le conseil. Il montre que la nature de l'interaction entre conseiller et producteur oriente le processus de décision du producteur, mais aussi que le profil du conseiller influe fortement sur la nature et l'intensité de cette interaction. Ingram (2008) souligne aussi la diversité des pratiques professionnelles entre des conseillers travaillant sur un même programme d'amélioration des pratiques agricoles : certains sont impliqués dans des relations asymétriques avec les agriculteurs générant des tensions et un manque de confiance, tandis que d'autres parviennent à stimuler des processus d'apprentissage pour une transition vers une agriculture plus durable. Cependant, les conseillers apprennent des difficultés qu'ils rencontrent dans leur situation de conseil et font évoluer leur cadre de réflexions (Guillot et al., 2013). De manière générale, les conseillers, selon leurs caractéristiques personnelles, la formation qu'ils ont eue, la trajectoire professionnelle qu'ils ont suivie, et selon la représentation qu'ils se font de leur métier, remplissent leurs fonctions de manière différente (Rémy et al., 2006).

L'objectif de l'article est d'analyser l'influence de la représentation que se font les conseillers de leur métier sur leurs pratiques professionnelles, dans le cadre d'un conseil qui s'appuie sur des méthodes standardisées. Nous faisons l'hypothèse que les représentations des conseillers sont fortement influencées par leur profil professionnel. Ces représentations entraînent une forte diversité des pratiques de conseil qui l'emporte sur l'objectif de standardisation du conseil affiché par les organisations 
de conseil. Nous analysons une expérience de mise en œuvre du Conseil à l'exploitation agricole familiale (CEF) au Bénin. L'expérience du CEF est intéressante parce qu'elle se caractérise par (i) des formes particulières d'interactions entre le conseiller et l'agriculteur visant à favoriser l'apprentissage dans le domaine de la gestion de l'exploitation, et (ii) la mise en œuvre d'une méthode de conseil standardisée afin de permettre à des conseillers issus de différents horizons professionnels de fournir un service homogène à un grand nombre d'agriculteurs. De plus, dans le cas du Bénin, le CEF est mis en œuvre depuis plus de deux décennies; ce qui permet de donner à l'analyse une profondeur historique. Cette étude est une contribution aux recherches conduites sur les conseillers qui exercent leur métier dans des situations de rationalisation des nouvelles approches de conseil. Les résultats devraient être utiles pour améliorer les dispositifs de conseil qui s'appuient sur des méthodes standardisées.

\section{Le Conseil à l'exploitation agricole familiale au Bénin}

Le système « Formation et Visites » (Anderson et al., 2006) promu par la Banque mondiale a constitué pendant plus de deux décennies la pierre angulaire du dispositif de vulgarisation agricole publique au Bénin. Les services déconcentrés de l'État fournissaient un ensemble de prestations aux agriculteurs (information et formation sur les techniques agricoles, offre de crédit, vente d'intrants, appui à la commercialisation de certains produits comme le coton). La restructuration des services agricoles intervenue en 1992, pour faire suite au Projet de restructuration des services agricoles du ministère de l'Agriculture, a réduit la mission des services publics aux seules fonctions de conseil ; et elle a rendu possible l'implication d'acteurs autres que publics dans le conseil agricole (Tossou, 1996 ; Moumouni et al., 2011). Actuellement, la Stratégie nationale de mise en œuvre du conseil agricole (SNCA) reconnaît l'importance de favoriser l'intervention d'une diversité de prestataires de services de conseil et de démarches de conseil (MAEP, 2008). Ainsi, aujourd'hui, plusieurs organisations de producteurs et ONG fournissent un conseil aux agriculteurs et le CEF est reconnu par nombre de ces acteurs comme une démarche pertinente. Le CEF est une démarche globale de renforcement des capacités des producteurs et de leurs familles, qui prend en compte les aspects techniques, économiques, sociaux et environnementaux de l'activité agricole (Faure et al., 2004). Il repose sur des méthodes d'apprentissage et d'aide à la décision qui permettent aux adhérents de mieux rationaliser leurs décisions. Il vise à initier les agriculteurs à la gestion dans le cadre d'un cycle comprenant différentes phases (analyse, programmation, décision/action, suivi, et évaluation).

Les agriculteurs bénéficiant des divers services de conseil béninois ont des profils variés (âge, sexe, niveau d'instruction, occupation principale, etc.) et disposent d'exploitations différenciées (type de productions, superficies emblavées...). Mais ils sont en majorité des petits producteurs (de un à quelques hectares) produisant à des degrés divers pour l'alimentation de leur famille et le marché. Les agriculteurs qui accèdent au CEF sont également des petits producteurs, mais possèdent souvent des exploitations mieux dotées en ressources que la moyenne des agriculteurs et orientent une plus forte part de leur production vers les marchés (Faure et al., 2004).

Le CEF est mis en œuvre dans le cadre de différents projets soutenus par la coopération internationale et notamment dans le cadre du Programme d'appui aux dynamiques productives (PADYP), financé par l'Agence française de développement 
(AFD), qui a débuté en 2008. Ce projet fait suite à d'autres projets qui ont permis d'expérimenter et de faire évoluer la méthode de CEF depuis 1998. Plusieurs organisations mettent en œuvre le CEF au Bénin, dont la Fédération des unions des producteurs (FUPRO), depuis 2010. Les conseillers organisent des séances de conseil en groupe et assurent aussi un suivi individualisé des agriculteurs. Après une phase de diagnostic des exploitations permettant d'identifier les demandes et d'orienter les actions, ils organisent des formations collectives sur les techniques agricoles et sur les concepts et outils de gestion (calcul de la marge brute et du prix de revient, plan prévisionnel de campagne, gestion des récoltes, compte recettes-dépenses, etc.). Les conseillers peuvent être amenés à conduire des actions d'alphabétisation. Ils peuvent aussi appuyer la conception et l'exécution de projets individuels ou collectifs (achat d'intrants, commercialisation de produits, etc.). Le CEF favorise ainsi le raisonnement des agriculteurs, les amène à intégrer la prévision dans leurs pratiques et à utiliser des indicateurs technico-économiques dans l'analyse de leurs résultats. Dans le cadre du CEF, des échanges entre producteurs sont encouragés à travers différentes actions collectives (formations, réunions de groupe pour discuter les résultats, visites au champ pour partager des expériences, essais dans les parcelles paysannes pour tester des innovations...).

Dans le cadre du PADYP, chaque conseiller a en charge l'animation directe de deux groupes de producteurs d'au moins une trentaine d'adhérents chacun. Il a également en charge la création et le suivi de sept autres groupes pilotés par des animateurs paysans. Les animateurs paysans réalisent certaines actions d'animation et des suivis individualisés des agriculteurs qui participent au CEF dans leur village. La majorité des groupes sont constitués d'agriculteurs alphabétisés tandis que certains regroupent des non-alphabétisés. Les activités du conseiller sont définies dans un cahier des charges élaboré par le PADYP qui codifie de manière précise à la fois les activités de conseil et la conduite des relations avec les participants et les animateurs paysans (Bernard et al., 2010). Les conseillers de la FUFRO ont reçu une même formation préalablement à leur intervention sur le terrain : maîtrise des techniques de production et des concepts de gestion de l'exploitation, maîtrise des outils de gestion, conduite de la relation de conseil, définition des étapes d'intervention (Daniel-Clarys, 2011). Cette formation a été dispensée par le PADYP et certains cadres de la FUPRO. La formation et le cahier des charges ont pour objectifs de construire une représentation collective du CEF chez les conseillers et d'assurer une standardisation du service fourni aux agriculteurs.

\section{Cadre d'analyse et méthode}

Les représentations que les conseillers ont de leur rôle et de leur position dans l'organisation du conseil reposent sur leurs pratiques de conseil. Selon Abric (2001), la représentation sociale est un mécanisme d'interprétation de la réalité qui régit les relations des individus à leur environnement et qui détermine leurs pratiques et actions. Elle résulte de processus sociaux impliquant l'individu à travers son histoire et son vécu, ainsi qu'à travers son insertion et dans un système social. La théorie de la représentation sociale dite du «noyau central » postule qu'une représentation sociale se structure autour d'un noyau central qui détermine sa structure, sa signification et son fonctionnement. Le noyau central regroupe les composantes les plus stables et les mieux acceptées par les membres du groupe social. Il comprend ici la compréhension des principes du CEF (diagnostic des exploitations agricoles, mobilisation d'outils de gestion, tâches des conseillers) qui est commune à tous les conseillers et qui permettrait une mise en œuvre 


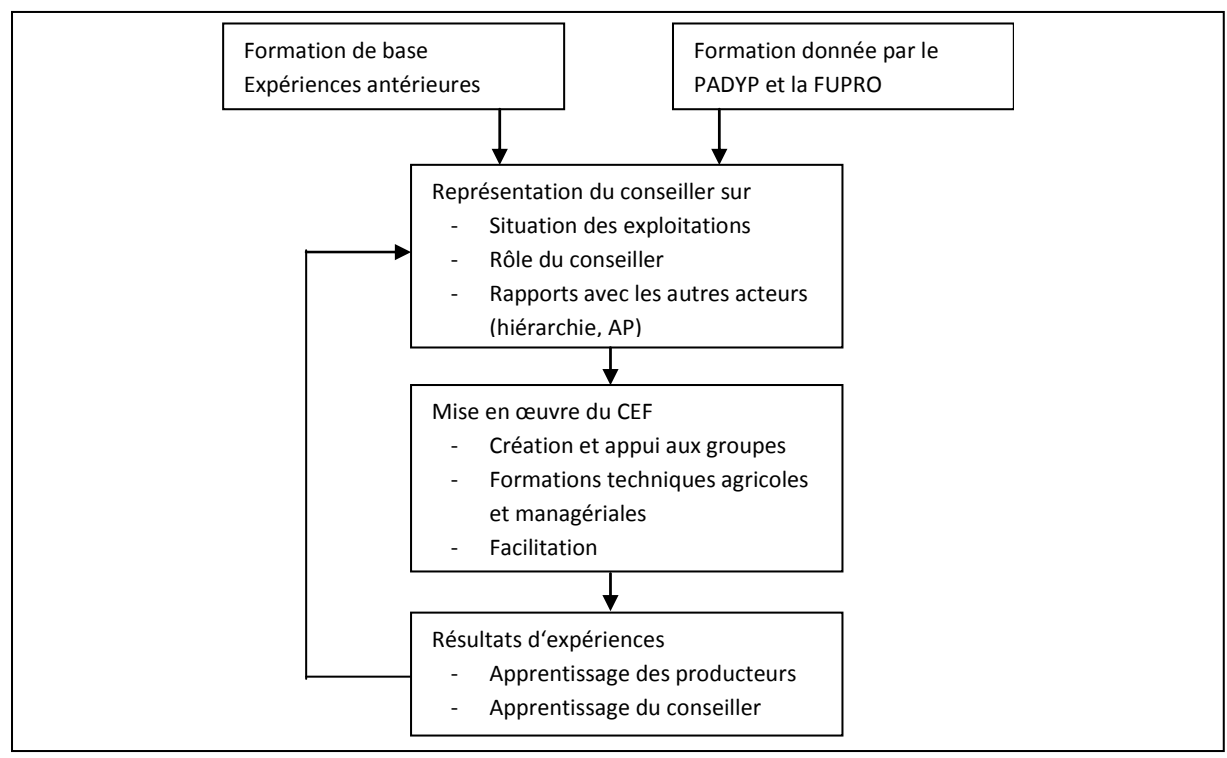

Source : les auteurs.

standardisée du conseil. Les formations données par le PADYP et les cadres de la FUPRO aux conseillers visent à asseoir un tel noyau central. Au noyau central d'une représentation sociale se rattachent des composantes périphériques (Abric, 2003). Ces éléments périphériques sont plus souples, plus adaptatifs et rendent possibles les réajustements quotidiens que nécessitent les situations (Flament, 1994). Dans cet article, nous nous focalisons sur ces éléments périphériques pour expliquer les différences individuelles dans la mise en œuvre du conseil. La théorie des principes organisateurs (Doise et al., 1990) réfute cependant l'idée que l'étude des représentations sociales pourrait se limiter aux éléments de ces représentations qui sont communs aux membres d'un groupe. Des différences interindividuelles de représentation sociale sont en jeu et proviennent des caractéristiques individuelles (genre, âge, statut, niveau d'instruction, niveau de prospérité, etc.) et de l'appartenance des acteurs à des groupes sociaux. Ces facteurs individuels expliquent pourquoi, bien qu'ils soient supposés partager une représentation commune du CEF, les conseillers ont une représentation personnelle formée d'opinions et de croyances particulières sur ce que sont la situation et les besoins des exploitations, sur ce que le CEF peut apporter aux producteurs, sur leur rôle de conseiller. Ces représentations des conseillers influencent les modalités d'action qui produisent le conseil (création de groupes et appui aux groupes, formations aux techniques agricoles et managériales, suivi individuel des exploitations, travail de facilitation). La mise en œuvre de ces activités génère un processus d'apprentissage chez les producteurs, mais aussi chez les conseillers. Un tel ajustement permanent entre représentations et expériences, révélateur d'une certaine réflexivité, est nécessaire pour un apprentissage effectif (Mezirow, 1997 ; Percy, 2005). La figure 1 résume ce cadre d'analyse. 
L'étude que nous présentons a été menée en 2011 et a concerné les seize conseillers de la FUPRO en activité dans les différentes communes de deux régions (Ouémé, Plateau). Ces régions ont été choisies parce que l'usage du CEF y est plus fortement développé que dans les autres régions. Les exploitations sont en culture manuelle, de petite taille, cultivant principalement du maïs et du manioc, et dans une moindre mesure des plantes légumineuses et du coton. Les données collectées se rapportent (i) aux caractéristiques des conseillers en termes d'expérience et de mise en œuvre du CEF, (ii) à leurs représentations du CEF (pertinences, points forts et faibles), des problèmes à résoudre avec les agriculteurs et de leur rôle de conseiller, (iii) à leurs activités et aux modalités de mise en œuvre du conseil (diagnostic réalisé, formations données, outils de gestion utilisés, thématiques abordées, suivis individuels réalisés, projets individuels ou collectifs accompagnés). Nous avons collecté ces données grâce à des entretiens semi-directifs menés auprès des responsables de la FUPRO en charge du conseil agricole, des seize conseillers, des animateurs-paysans et des producteurs participant au CEF. Ces données d'entretien ont été complétées par celles provenant des observations effectuées lors de notre participation à certaines séances de formation organisées par les conseillers. L'analyse de discours s'est faite en trois temps. Dans un premier temps, le discours de chaque conseiller a été examiné afin de repérer et caractériser ses représentations de l'exploitation familiale et les difficultés de cette dernière, du CEF, du rôle du conseiller. Ensuite, en comparant les différentes représentations des conseillers, nous avons effectué une catégorisation de ces dernières qui nous a permis d'identifier des types de conseillers. Des extraits de discours des participants au CEF, numérotés de $\mathrm{A} 1$ à A16, sont présentés dans l'article en italique et entre guillemets pour illustrer certaines analyses. Enfin, de cet exercice de catégorisation des représentations, ont émergé des thématiques qui sont discutées.

\section{Résultats}

Les résultats sont présentés en cinq soussections. Dans un premier temps, nous présentons quelques données socioéconomiques globales sur les conseillers (section 1). Nous présentons ensuite les représentations des conseillers, issues d'une analyse de leurs discours recueillis lors des entretiens individuels (section 2), puis les modalités concrètes de leurs actions (nombre de groupes conseillés, part du conseil individuel, implication dans l'expérimentation...) identifiées par l'observation des conseillers en action (section 3). Cette description des pratiques est étayée par des entretiens avec les managers des conseillers et les animateurs-paysans, ainsi que par des observations participantes. Nous croisons ensuite représentations et modalités d'action des conseillers (section 4), afin d'établir trois types de conseillers dont nous décrivons les caractéristiques socio-économiques (section 5). Cette dernière section permet de vérifier qu'il y a bien un lien entre l'appartenance des agriculteurs à des groupes sociaux, leur représentation du conseil et les modalités de mise en œuvre des services.

\section{Qui sont les conseillers ?}

Les conseillers ont des profils socioéconomiques différents (tableau 1). Majoritairement hommes, ils ont entre 26 et 45 ans. Ils ont tous une formation équivalente ou supérieure au Bac dans le domaine agricole. Tous les conseillers ont eu au moins une expérience professionnelle préalable avant d'occuper leur poste actuel, condition préalable à leur recrutement. Par contre, leurs parcours professionnels sont variés. Par exemple, l'un d'entre eux a d'abord été producteur agricole. Il est ensuite devenu animateur-paysan, puis 
conseiller. Un autre, ingénieur agronome, a 16 ans d'expérience dont 10 dans le CEF. Ces trajectoires et ces expériences professionnelles différentes jouent un rôle important dans la vision que les conseillers ont du milieu agricole et de la manière dont il faut travailler avec les producteurs.

Pour l'ensemble des conseillers qui ont fait l'objet d'enquêtes, un bon conseiller doit avoir de solides compétences techniques en agriculture et en gestion, ainsi qu'une formation ou une expérience en milieu rural. Du point de vue de la motivation, ils évoquent presque tous le « goût du métier », car le métier de conseiller demanderait un certain niveau d'engagement. Les conseillers qui ont un niveau d'instruction supérieur aux autres conseillers sont aussi ceux qui sont le plus expérimentés dans l'utilisation des techniques d'animation du CEF ou du conseil en milieu rural en général. Ceci est en partie dû à la politique de recrutement des projets. Le PADYP exige un niveau de formation de base moins élevé que le projet qui l'a précédé. De même, avec l'expérience, les conseillers déclarent prendre davantage conscience de la complexité de l'exploitation agricole familiale, élargir les horizons de leur diagnostic d'exploitation et ajuster les activités mises en œuvre en conséquence.

\section{Quelles sont les représentations des conseillers ?}

Nous avons analysé le discours des conseillers pour caractériser, d'une part, leur représentation des difficultés rencontrées par les producteurs dans la conduite de leur exploitation et, d'autre part, leur représentation du rôle qu'ils jouent.

\section{Les difficultés rencontrées} par les producteurs

Trois types de discours sont ressortis concernant le diagnostic sur les difficultés auxquelles les producteurs font face et qui

Tableau 1. Caractéristiques des conseillers

\begin{tabular}{l|l|l}
\hline Caractéristiques des conseillers & Attributs/paramètres & Valeurs \\
\hline Sexe & Femme & $12 \%$ \\
Âge & Homme & $88 \%$ \\
& Moyenne & 33 \\
Niveau de formation & Minimum & 26 \\
Domaine de formation & Maximum & 45 \\
& Diplôme d'études d'agriculture tropicale (Bac) & $69 \%$ \\
Années d'expérience en agriculture & Brevet de technicien supérieur ou plus (BTS ou ingénieur) & $31 \%$ \\
& Production animale ou végétale & $37 \%$ \\
& Vulgarisation et gestion & $19 \%$ \\
Années d'expérience en animation & $44 \%$ \\
& Autres (génétique, foresterie et génie civil) & 08 \\
Années d'expérience en CEF & Minimum & 03 \\
& Maximum & 20 \\
& Moyenne & 07 \\
& Minimum & 02 \\
& Maximum & 20 \\
& Moyenne & 03 \\
& Minimum & 01 \\
\hline
\end{tabular}


doit donc donner lieu à du conseil. Tout d'abord, certains conseillers évoquent seulement des difficultés techniques et financières (baisse de la fertilité des sols, nonmaîtrise de l'eau et des ravageurs, manque d'appui technique, manque de capital et de trésorerie, etc.). D'autres évoquent ces éléments, mais mettent surtout l'accent sur les difficultés d'accès aux ressources et aux marchés (difficultés d'accès aux intrants de qualité, accès limité aux ressources foncières, pénurie de main-d'œuvre salariée, difficulté d'écoulement des produits agricoles...). Enfin, les conseillers du dernier groupe, même s'ils sont conscients d'autres types de difficultés, évoquent surtout des problèmes de gestion des ressources disponibles au sein de l'exploitation ou d'organisations collectives des producteurs. Ce sont ces conseillers qui $a$ priori sont les plus concernés par la gestion de l'exploitation.

\section{Le rôle du conseiller}

L'analyse des entretiens avec les conseillers montre qu'ils diffèrent également dans leur manière de définir leur rôle, et parfois les compétences associées à ce rôle. Certains conseillers vont clairement définir leur rôle comme de la transmission de savoirs techniques et de gestion. Les conseillers « guident $»(\mathrm{~A} 5)$, « suivent $»$ ou « assistent» (A9) les producteurs dans leurs activités, aident les producteurs à «corriger leurs erreurs » (A2) ou à « surmonter leurs difficultés en les formant sur des aspects techniques et de gestion » (A6). Ils les «forment aux outils de gestion» (A2), les «aident sur les thématiques qu'ils ont identifiées dans le diagnostic de l'exploitation agricole » (A16). Même si certains de ces conseillers disent vouloir " guider » les producteurs, ou les « orienter et les aider à atteindre les objectifs qu'ils se sont fixés », ils sont dans une posture prescriptive, de détenteurs des savoirs qu'ils doivent «transmettre » (A13) aux agriculteurs. Ces conseillers ont une posture qui rappelle celle de la vulgarisation agricole et d'encadrement des producteurs même si les éléments transférés ne sont pas seulement des recommandations techniques, mais aussi des outils de gestion.

D'autres conseillers ont un discours plus orienté vers l'aide à la décision pour la gestion de l'exploitation, en « analysant avec les producteurs leur situation afin de leur permettre de prendre des décisions concrètes »(A1). Par exemple, un conseiller dit vouloir aider les producteurs « à prendre des décisions pertinentes en vue d'augmenter son revenu» (A10). Ils parlent aussi d' " accompagner les producteurs dans la définition des besoins, dans le suivi de leurs résultats techniques et économiques, dans la mise à disposition d'outils de planification, dans la recherche de moyens nécessaires pour exécuter leurs activités » (A7). Le discours de ces conseillers suggère une volonté d'accompagnement et d'appui à un processus dans lequel le producteur doit être pris en compte.

Enfin, des conseillers évoquent ces postures d'aide à la décision pour la gestion de l'exploitation, mais en y ajoutant la fonction de facilitation et d'intermédiation que, selon eux, le conseiller doit aussi avoir. Certes le conseiller appuie les producteurs et les amène à analyser leurs situations, mais c'est aussi « un facilitateur qui permet aux producteurs de trouver des solutions à tous les problèmes et dans tous les domaines »(A3). Le conseiller est ainsi «celui qui fait tout : il est à la fois confident, facilitateur et formateur » (A4). Il « amène les producteurs à mieux gérer leurs exploitations agricoles en les appuyant sur les aspects techniques, en les formant sur les aspects de gestion de l'exploitation, en induisant et facilitant leur contact avec les institutions locales (microcrédit, par exemple), et en les aidant autant qu'il peut à trouver des solutions aux problèmes quotidiens » (A12). Il n'a pas forcément besoin d'avoir toutes les 
connaissances nécessaires pour apporter des conseils puisqu'il « fait appel à des compétences externes pour répondre aux besoins de ses producteurs »(A8). Des aspects plus personnels de la relation au producteur et à sa famille ressortent dans le discours de ces conseillers. Pour l'un d'entre eux, par exemple, le conseiller doit « aider à l'épanouissement des producteurs sur les plans du revenu, social et familial » (A14).

L'enquête montre qu'il existe une relation entre la manière dont les conseillers perçoivent les difficultés des exploitations et la représentation qu'ils ont de leur rôle. Les conseillers qui considèrent que leur rôle est plutôt dans la transmission de connaissances techniques et de gestion ont un diagnostic des difficultés de l'exploitation qui met en avant les contraintes techniques et financières, ainsi que les contraintes externes à l'exploitation. Ceux qui considèrent que leur rôle est plutôt dans la facilitation et l'aide à la décision pour la gestion de l'exploitation ont un diagnostic plus large des difficultés des exploitations.

Finalement, l'analyse des représentations des conseillers nous permet de dégager trois types de conseillers en fonction de ce qu'ils pensent être : des conseillers « vulgarisation de techniques agricoles et de concepts de gestion », des conseillers « appui à la gestion et aide à la décision » et des conseillers « appui à la gestion, intermédiation et facilitation ». Nous décrivons en détail ces trois types plus loin.

\section{Quelle est la variabilité des modalités d'action des conseillers}

Les conseillers, bien qu'ayant reçu un cahier des charges identique et une même formation dans le cadre du programme, ne mettent pas en œuvre les activités de conseil au même rythme ni de la même manière. Ceci apparaît dans les entretiens, mais nous avons aussi pu le vérifier par une méthodologie d'observation des pratiques concrète du CEF. Le nombre de groupes supervisés par conseiller est une variable clé de la nature du service et de son niveau de standardisation. Or on constate à ce niveau d'importantes différences entre conseillers. Le cahier des charges précise que neuf groupes doivent être mis en place par conseiller. Or certains conseillers n'ont que huit groupes, car ils estiment devoir attendre que les groupes soient stabilisés pour en constituer d'autres. D'autres ont mis en place jusqu'à onze groupes, souvent parce que la demande de création de groupe de la part des agriculteurs est forte et qu'ils souhaitent y répondre. En ce qui concerne les activités de conseil, tous les conseillers commencent par des actions d'alphabétisation dans les groupes de producteurs non alphabétisés, déploient des formations aux techniques agricoles et mettent en place des activités de sensibilisation à la gestion des exploitations (formation à la tenue des cahiers et fiches, compréhension des concepts de gestion). Une majorité de conseillers s'est engagée dans une véritable démarche de gestion, qui nécessite que chaque participant mobilise ses propres relevés de données pour gérer son exploitation dans le cadre d'un cycle complet de gestion. Certains conseillers s'investissent beaucoup dans le diagnostic initial des exploitations agricoles et dans des formations aux techniques agricoles. Les parcelles de démonstrations sont un des éléments centraux de cette dimension du conseil. Or le nombre de parcelles de démonstration installées par les conseillers varie énormément, de 0 à 40 . Certaines activités, comme l'identification de projets individuels et collectifs, et l'intermédiation, ne sont mises en œuvre que par une petite minorité de conseillers. Enfin, la part de temps dédiée au suivi individuel des producteurs, tant pour fournir un appui technique que pour des conseils de gestion, est très variable d'un conseiller à l'autre (de quelques jours par mois à plus de la moitié du temps de travail). 


\section{Quelle relation entre les représentations et les modalités d'action des conseillers ?}

Il existe une relation forte entre les modalités d'action des conseillers et leur représentation des difficultés rencontrées par les exploitations et de leur rôle de conseiller. L'influence des représentations se traduit par exemple par l'importance relative très variable qu'ils accordent dans leur activité aux fonctions de transmission de connaissances, d'aide à la gestion ou d'intermédiation. Le tableau 2 met en évidence les différences de modalités d'action en fonction des représentations pour les trois types de conseillers que nous avons identifiés.

Les principales caractéristiques des trois types sont les suivantes:

\section{Type 1 : Conseillers « vulgarisation} de techniques agricoles et de concept de gestion »

Pour ces conseillers, les principales difficultés des producteurs sont d'ordre technique et financier, les aspects techniques étant plus particulièrement soulignés. Quoique certains d'entre eux évoquent une posture de « guide», ou d' " orientation » des producteurs, leur rôle en tant que conseiller est d'aider les producteurs à travers la formation technique et le transfert de connaissances sur la production agricole. Les outils de gestion sont enseignés aux producteurs, mais sans chercher à donner forcément du sens à leur utilisation. Les activités privilégiées sont la formation

Tableau 2. Les modalités d'action des conseillers en fonction de leur représentation

\begin{tabular}{|c|c|c|c|c|}
\hline \multicolumn{2}{|c|}{$\begin{array}{l}\text { Critères d'analyse et } \\
\text { de comparaison des } \\
\text { conseillers agricoles }\end{array}$} & \multirow{2}{*}{$\begin{array}{l}\text { Conseiller « vulgarisation } \\
\text { de techniques agricoles et } \\
\text { de concept de gestion " }\end{array}$} & \multirow{2}{*}{\begin{tabular}{|l|}
$\begin{array}{l}\text { Conseiller « appui à } \\
\text { la gestion et aide à la } \\
\text { décision " }\end{array}$ \\
Alphabétisation, \\
Sensibilisation à la \\
gestion, Formation \\
à la gestion, Suivi \\
technique et de gestion \\
des exploitations \\
individuelles \\
\end{tabular}} & \multirow{2}{*}{$\begin{array}{l}\text { Conseiller « appui à la } \\
\text { gestion et intermédiation } \\
\text { et facilitation " } \\
\text { Alphabétisation, } \\
\text { Sensibilisation à la gestion, } \\
\text { Formation à la gestion, } \\
\text { Identification de projets } \\
\text { individuels ou collectifs, } \\
\text { Intermédiation }\end{array}$} \\
\hline Activités & $\begin{array}{l}\text { Activités } \\
\text { principales }\end{array}$ & & & \\
\hline & $\begin{array}{l}\text { Activités } \\
\text { secondaires } \\
\text { ou absentes }\end{array}$ & $\begin{array}{l}\text { Identification de projets } \\
\text { individuels ou collectifs, } \\
\text { Formation à la gestion, } \\
\text { Intermédiation }\end{array}$ & \begin{tabular}{|l|} 
Identification de projets \\
individuels ou collectifs, \\
Intermédiation
\end{tabular} & \\
\hline & $\begin{array}{l}\text { Activités à } \\
\text { importance } \\
\text { variable }\end{array}$ & $\begin{array}{l}\text { Diagnostic initial des } \\
\text { exploitations, Formation } \\
\text { à la gestion }\end{array}$ & $\begin{array}{l}\text { Diagnostic initial } \\
\text { des exploitations, } \\
\text { Formation aux } \\
\text { techniques agricoles }\end{array}$ & $\begin{array}{l}\text { Diagnostic initial des } \\
\text { exploitations, Formation } \\
\text { aux techniques agricoles, } \\
\text { Suivi individuel des } \\
\text { exploitations } \\
\end{array}$ \\
\hline \multirow[t]{2}{*}{$\begin{array}{l}\text { Représen- } \\
\text { tations }\end{array}$} & \begin{tabular}{|l|} 
Difficultés \\
des \\
exploitations
\end{tabular} & Techniques et financières & $\begin{array}{l}\text { Techniques et } \\
\text { financières, } \\
\text { De gestion de } \\
\text { l'exploitation } \\
\end{array}$ & $\begin{array}{l}\text { Techniques et financières, } \\
\text { De gestion de l'exploitation, } \\
\text { D'accès aux ressources et } \\
\text { aux marchés }\end{array}$ \\
\hline & $\begin{array}{l}\text { Rôles de } \\
\text { conseiller }\end{array}$ & $\begin{array}{l}\text { Transmission de } \\
\text { connaissance technique } \\
\text { et de gestion } \\
\end{array}$ & $\begin{array}{l}\text { Aide à la décision } \\
\text { pour la gestion de } \\
\text { l'exploitation }\end{array}$ & $\begin{array}{l}\text { Facilitation et aide à la } \\
\text { décision pour la gestion de } \\
\text { l'exploitation }\end{array}$ \\
\hline \multicolumn{2}{|c|}{$\begin{array}{l}\text { Nombres et pourcentages } \\
\text { de conseillers concernés }\end{array}$} & $6(38 \%)$ & $6(38 \%)$ & $4(25 \%)$ \\
\hline
\end{tabular}


en salle et les visites des parcelles d'essai. L'appui individualisé sur le terrain peutêtre important, mais est principalement réservé au suivi des itinéraires techniques des parcelles. Dans ce cas de figure, le conseiller adopte beaucoup plus une posture d'enseignant dont l'animateur paysan est le relais pour transmettre des informations et des connaissances auprès des producteurs.

\section{Type 2 : Conseiller " appui à la gestion et aide à la décision »}

Les conseillers de ce type estiment qu'en plus des difficultés techniques, les producteurs sont aussi confrontés à des problèmes d'accès aux ressources et aux marchés. Leur vision de l'exploitation va au-delà de la prise en compte des aspects liés aux techniques agricoles. En conséquence, ils voient leur rôle comme un accompagnement et un appui aux processus de prise de décision des producteurs, pour améliorer la gestion de leur exploitation. L'utilisation des outils de gestion est au cœur du métier du conseiller pour mettre en place une démarche de planification des activités de l'exploitation, de suivi et d'évaluation. La formation en salle est également une activité centrale pour ce type de conseiller alors que la mise en place de parcelles expérimentales est peu importante. Le suivi individualisé permet d'aborder à la fois des questions liées aux techniques agricoles, mais aussi à la gestion des ressources de l'exploitation. Certains conseillers de ce groupe perçoivent leur rôle comme central, mais insuffisamment récompensé. Le rôle de l'animateur-paysan est défini comme une aide au conseiller en servant d'intermédiaire avec les agriculteurs. Ce type de conseiller propose des formations techniques beaucoup plus managériales qu'agricoles et a souvent une bonne capacité d'écoute, de communication et d'animation.

\section{Type 3 : Conseiller « intermédiation et facilitation »}

Le diagnostic posé par les conseillers de ce type sur les exploitations agricoles va audelà des difficultés techniques et de gestion pour couvrir des difficultés d'accès aux intrants, aux ressources, et aux marchés. Ils évoquent de ce fait l'intermédiation comme le cœur de leur métier de conseiller. Comparée à celle du type 2 , la vision de l'exploitation agricole devient encore plus globale, car elle prend en compte l'environnement de l'exploitation. Non seulement les interactions entre les producteurs eux-mêmes sont importantes, mais ils considèrent que les producteurs doivent pouvoir être en contact avec d'autres acteurs de développement agricole susceptibles de leur offrir un service ou une opportunité de développement. Tout en mettant un accent fort sur la formation en salle des producteurs à la gestion des exploitations, ces conseillers consacrent un temps appréciable à l'identification de projets individuels ou collectifs et à des activités d'intermédiation pour améliorer l'accès à différents services pour les producteurs. Les parcelles expérimentales sont très peu développées et le suivi individualisé, pour appuyer la conduite technique et la gestion de l'exploitation, est très variable. Tout comme le conseilleur du type 2 , celui-ci a une bonne capacité d'écoute, de communication et d'animation.

\section{Quelle relation entre les caractéristiques socioéconomiques et les types d'appartenance des conseillers ?}

Le tableau 3 présente les caractéristiques socio-économiques des trois types de conseillers. Le type 1 se caractérise par une forte présence de jeunes conseillers avec un faible niveau de diplôme (titulaires d'un DEAT en vulgarisation et en gestion) et ayant moins de six ans d'expérience en agriculture et moins trois ans dans le CEF. Dans le type 2, on retrouve des conseillers 
plus âgés et plus expérimentés en agriculture que ceux du type 1, mais pas plus expérimentés en CEF. Ils ont souvent une formation initiale, spécialisés dans d'autres domaines (génétique, foresterie, génie rural). Dans le type 3, on note la présence des conseillers les plus âgés, diplômés et expérimentés dans l'agriculture et la mise en œuvre du CEF. Ces résultats montrent qu'en fonction du niveau initial de formation, mais surtout de leur expérience dans l'agriculture et le CEF, les conseillers développent une autre représentation des besoins des agriculteurs et de leur rôle : ils dépassent les appuis techniques et de gestion et incluent des appuis pour faciliter l'accès à divers services agricoles.

\section{Discussion}

De l'analyse des discours des conseillers sur différents aspects de leur métier, nous avons identifié trois types de conseillers à savoir des conseillers "vulgarisation de techniques agricoles et de concepts de gestion », des conseillers « appui à la gestion et aide à la décision » et les conseillers « intermédiation et facilitation ». Ces résultats montrent à quel point les représentations du conseiller influencent sa pratique du conseil agricole et sa posture vis-à-vis des producteurs participant au conseil.

Ces résultats corroborent ceux de Rémy et al. (2006) sur le fait que, malgré une relative standardisation de la méthode de conseil et une formation identique, les conseillers mettent différemment en œuvre le CEF, en fonction de leurs profils socio-économiques et socioprofessionnels et de la représentation qu'ils se font de leur métier. Compagnone (2001) a analysé l'organisation du conseil au sein de l'Institut de l'Élevage en France et l'identité de cette organisation et de ses ingénieurs. Il montre que la programmation des activités de conseil dépend plus des choix faits par chaque ingénieur que de ceux énoncés par les responsables de l'institut, car ces ingénieurs se sentent plus redevables vis-à-vis de leur public que de leur hiérarchie. Ces résultats montrent aussi comment, dans les mêmes conditions professionnelles

Tableau 3. Caractéristiques socio-économiques des trois types de conseillers

\begin{tabular}{|l|l|c|c|c|}
\hline Caractéristiques & Attributs & $\begin{array}{c}\text { Conseiller } \\
\text { « vulgarisation de } \\
\text { techniques agricoles et } \\
\text { de concept de gestion » } \\
\%(6 \text { conseillers) }\end{array}$ & $\begin{array}{c}\text { Conseiller } \\
\text { "appui à la } \\
\text { gestion et aide } \\
\text { à la décision » } \\
\%(6 \text { conseillers) }\end{array}$ & $\begin{array}{c}\text { Conseiller } \\
\text { " intermédiation } \\
\text { et facilitation » } \\
\%(4 \text { conseillers) }\end{array}$ \\
\hline \multirow{2}{*}{ Sexe } & Femme & $17(1)$ & $00(0)$ & $25(1)$ \\
\cline { 2 - 5 } & Homme & $83(5)$ & $100(6)$ & $75(3)$ \\
\hline \multirow{2}{*}{ Âge } & Moins de 30 ans & $67(4)$ & $50(3)$ & $00(0)$ \\
\cline { 2 - 5 } & Plus de 30 ans & $33(2)$ & $50(3)$ & $100(4)$ \\
\hline \multirow{2}{*}{ Diplôme } & DEAT & $83(5)$ & $83(5)$ & $25(1)$ \\
\cline { 2 - 5 } & BTS & $17(1)$ & $17(1)$ & $75(3)$ \\
\hline \multirow{2}{*}{$\begin{array}{l}\text { Domaine de } \\
\text { formation }\end{array}$} & Vulgarisation et gestion & $50(3)$ & $33(2)$ & $25(1)$ \\
\cline { 2 - 5 } & Production vég. ou anim. & $33(2)$ & $33(2)$ & $50(2)$ \\
\cline { 2 - 5 } & Autres & $17(1)$ & $33(2)$ & $25(1)$ \\
\hline \multirow{2}{*}{$\begin{array}{l}\text { Expérience en } \\
\text { agriculture }\end{array}$} & Nouveau (moins de 6 ans) & $83(5)$ & $33(2)$ & $00(0)$ \\
\cline { 2 - 5 } & Ancien & $17(1)$ & $67(4)$ & $100(4)$ \\
\hline \multirow{2}{*}{$\begin{array}{l}\text { Expérience dans } \\
\text { le CEF }\end{array}$} & Nouveau (moins de 3 ans) & $83(5)$ & $83(5)$ & $25(1)$ \\
\cline { 2 - 5 } & Ancien & $17(1)$ & $17(1)$ & $75(3)$ \\
\hline
\end{tabular}


(cahier des charges, formations reçues en préparation à l'exercice du poste, etc.), les conseillers s'inscrivent dans des registres d'action différents en fonction des représentations qu'ils ont de leur métier (objet du conseil, rôle du conseiller, rapports socioprofessionnels, etc.), mais aussi des possibilités d'action qui s'ouvrent à eux selon les situations. On peut donc dire que le conseiller, selon ses caractéristiques personnelles et celles de son milieu d'intervention, est constamment engagé dans un processus d'arbitrage entre ses propres représentations du conseil, ses attributions contractuelles définies par sa hiérarchie et la situation d'interaction dans laquelle il se trouve. Dans une situation donnée, les activités qu'il mène, les priorités qu'il définit et les choix qu'il effectue sont le reflet de ce compromis entre les exigences de son cahier des charges et les éléments de ses représentations. Ces résultats mettent en exergue la complémentarité de la théorie du noyau social (Abric, 2001) et celle des principes organisateurs (Doise, 1990). En plus, des éléments communs aux conseillers, il existe aussi des différences interindividuelles de représentations qui proviennent des caractéristiques personnelles des conseillers comme leur niveau de formation et leur expérience professionnelle.

$$
\begin{gathered}
* \\
* *
\end{gathered}
$$

D'après les résultats de cette étude, les conseillers exécutent de manière différente un même cahier des charges qui définit ce que doivent être le conseil et les méthodes et outils pour le mettre en œuvre. Le niveau de formation et l'expérience acquise au cours de leur trajectoire professionnelle influencent la représentation qu'ils ont de leur métier. Il n'est donc pas surprenant qu'ils aient des comportements différents en situation professionnelle alors qu'ils ont reçu une formation identique afin de maîtriser les méthodes et outils du CEF.
Ainsi, l'étude a permis d'identifier trois types de conseillers.

Au regard de ces résultats, on pourrait à juste titre s'interroger sur le fait qu'un type de conseiller soit «plus efficace » pour fournir un conseil de qualité. À première vue, on pourrait penser que le conseiller le plus efficace serait le facilitateur parce qu'il adopte une démarche plus globale et plus participative. En réalité, il semble que la posture d'un conseiller est la plus efficace quand elle est adaptée au contexte d'intervention, aux objectifs poursuivis par l'organisation de conseil, et aux demandes des paysans. Les trois types peuvent donc donner, selon les situations, des résultats satisfaisants tant du point de vue des producteurs que de l'organisation qui fournit le conseil. L'étude n'a pas cherché à caractériser ces aspects qui peuvent être appréhendés de manière diverse (évolution des performances des exploitations, évolution des savoirs, satisfaction des participants, etc.). Il peut être intéressant que le conseiller et son organisation d'appartenance soient conscients de l'existence d'une gamme variée de postures permettant de traiter de questions différentes. Étant donné que les expériences professionnelles influencent les représentations du conseiller, il convient d'avoir aussi à l'esprit que sa posture peut évoluer progressivement au cours du temps, le conseiller pouvant passer d'un type à un autre. Pour accompagner plus efficacement les conseillers dans l'exercice de leurs fonctions, les organisations de conseil peuvent chercher à identifier les éléments clés de la représentation qui peuvent évoluer plus ou moins rapidement, de ceux qui ne peuvent changer que très lentement, car faisant plus intimement partie de la personnalité du conseiller.

Cette diversité pourrait être mise à profit par les organisations du conseil en organisant les complémentarités et les synergies entre ces types différents au niveau d'un même territoire d'intervention. 
Pour améliorer la mise en œuvre du CEF, il faut certes se focaliser sur les besoins exprimés par les producteurs, mais il faut également tenir compte de la spécialisation, des compétences particulières et des représentations des conseillers. Le conseiller disposant d'une marge de manœuvre d'adaptation du conseil, il serait intéressant, comme suggéré par Djamen et al. (2010), de développer chez les conseillers, avec des démarches de formation adaptées, une plus grande capacité d'adaptation des méthodes de conseil. Ainsi, au regard de la diversité des représentations et des types des conseillers, il serait utile de réfléchir, aussi bien dans les organisations de conseil que dans celles en charge de la formation continue des conseillers, à la mise en place de plates-formes permettant des échanges entre ces agents. Ces échanges peuvent porter sur (i) les démarches, méthodes et outils du conseil et (ii) sur les expériences des conseillers, sur la manière dont ils analysent leurs activités et sur la manière de mieux répondre et de mieux adapter leur intervention aux besoins d'accompagnement des producteurs. Cela permettrait notamment de développer chez ces personnes une réflexivité sur leurs pratiques professionnelles.

\section{RÉFÉRENCES BIBLIOGRAPHIQUES}

Abric J.-C. (2001). Les représentations sociales : aspects théoriques. In J.-C. Abric, Pratiques sociales et représentations, Paris, Presses universitaires de France, pp. 11-35.

Abric J.-C. (2003). L'analyse structurale des représentations sociales. In S. Moscovisci, F. Buschini, Les méthodes des sciences humaines, Paris, Presses universitaires de France, pp. 375-392.

Andersen H. J. (2004). Different personal skills and competencies which local agricultural advisers can use to co-create change in management procedures: a case-study of Danish dairy farmers and advisers. Journal of Agricultural Education and Extension, $\mathrm{n}^{\circ} 10$, pp. 151-62.

Anderson J. R., Feder G., Ganguly S. (2006). The Rise and Fall of Training and Visit Extension: An Asian Mini-drama with an African Epilogue. World Bank Policy Research, Working Paper 3928, May.

Bernard T., Delarue J., de Romemont A., Faure G. (2010). Proposition technique pour l'évaluation des impacts du Conseil à l'Exploitation familiale au Bénin. Paris, AFD-CIRAD.
Cerf M., Hemidy L. (1999). Designing support to enhance co-operation between farmers and advisors in solving farm-management problems. The Journal of Agricultural Education and Extension, ${ }^{\circ}$ 6, pp. 157-170.

Compagnone C. (2001). Pratiques d'ingénieurs et identité de l'Institut de l'Élevage. Économie rurale, ${ }^{\circ}$ 262, pp. 76-91.

Daniel-Clarys C. (2011). Du conseil global aux agriculteurs à la formation pédagogique des conseillers/formateurs : proposition de méthodes et outils pour une formation des conseillers plus adaptée et plus performante. Rouen, Université de Rouen - UFR Sciences de l'homme et de la société.

Djamen P., Havard M., Wey J. (2010). Renouvellement de l'offre de conseil au Nord-Cameroun : le rôle déterminant du conseiller. Papier présenté au Colloque SFER, Conseil en agriculture : acteurs, marchés, mutations, Dijon, 14-15 octobre.

Doise W. (1990). Les représentations sociales. In R. Ghiglione, C. Bonnet, J.-F. Richard (dir.), Traité de psychologie cognitive, Paris, Dunod, pp. 111-174. 
RECHERCHE Ismail MOUMOUNI, Aurelle de ROMEMONT, Fortuné AMONSOU-BIAOU, GUy FAURE

Faure G., Dugue P., Beauval V. (2004). Conseil à l'exploitation familiale. Expériences en Afrique de l'Ouest et du Centre. Paris, Éditions GRET.

Faure G., Desjeux Y., Gasselin P. (2012). New challenges in agricultural advisory services from a research perspective: a literature review, synthesis and research agenda. Journal of Agricultural Education and Extension, vol. 18, n ${ }^{\circ}$ 5, pp. 461-492.

Flament C. (1994). Structure, dynamique et transformation des représentations sociales. In J.-C. Abric (dir.), Pratiques sociales et représentations, Paris, Presses universitaires de France, pp. 37-58.

Gadrey J. (1994). Relations, contrats et conventions de service. In J. De Bandt, J. Gadrey (dir.), Relations de service, marchés de service, Paris, CNRS.

Guillot M. N., Cerf M., Petit M. S., Olry P., Omon B. (2013). Développer la capacité des conseillers à agir face à la diversité des situations de conseil en grande culture. Économie rurale, $\mathrm{n}^{\circ} 337$, pp. 59-74.

Ingram J. (2008). Agronomist-farmer knowledge encounters: an analysis of knowledge exchange in the context of best management practices in England. Agriculture and Human Values, ${ }^{\circ} 25$, pp. 405-418.

Labarthe P., Laurent C. (2011). Service economics and public policies for agricultural extension. Cahiers Agricultures, vol. 20, ${ }^{\circ} 5$, pp. 343-351.

Lemery B. (1994). Lectures sociologiques des activités de conseil technique en agriculture.
Organisation et dynamiques. In J.-P. Darré (dir.), Pairs et experts dans l'agriculture, APAD-Karthala- IUED.

MAEP (2008). Stratégie nationale de mise en ceuvre du Conseil agricole au Bénin. Cotonou, Direction du conseil agricole et de la formation professionnelle, ministère de l'Agriculture, de l'Élevage et de la Pêche, MAEP.

Mezirow J. (1997). Transformative learning: Theory to practice. New Directions for Adult and Continuing Education, $\mathrm{n}^{\circ} 74$, pp. 5-12.

Moumouni M. I., Nouatin G., Baco M. N. (2011). Du système formation et visites au conseil à l'exploitation agricole familiale au Bénin : rupture ou continuité ? Cahiers Agricultures, $\mathrm{n}^{\circ}$ 20, pp. 376-81.

Percy R. (2005). The contribution of transformative learning theory to the practice of participatory research and extension: Theoretical reflections. Agriculture and Human Values $\mathrm{n}^{\circ} 22$, pp. 127-136.

Rémy J., Brives H., Lémery B. (2006). Conseiller en agriculture. Dijon, Éducagri Éditions, Quae Éditions.

Rogers E. M. (1988). The Intellectual Foundation and History of the Agricultural Extension Model. Science Communication, vol. $9, \mathrm{n}^{\circ} 4$, pp. 492-510.

Tossou C. R. (1996). Les acteurs et les réformes institutionnelles : une analyse de l'expérience béninoise en matière de professionnalisation. Série d'Économie et de Sociologie rurale, $\mathrm{n}^{\circ}$ 1996-05, FSA/UNB. AbomeyCalavi. 